\title{
Cap Sciences : un pont entre culture et économie
}

Étienne Guyon (Guyon@pmmh.espci.fr)

Physique et mécanique des milieux hétérogènes (PMMH), ESPCI, 10 rue Vauquelin, 75231 Paris Cedex 05.

\section{Construit en réhabilitant un}

des hangars qui bordaient la

Garonne en aval de la ville de

Bordeaux, un bâtiment lumi-

neux de 3600 mètres carrés, le

Hangar 20, abrite depuis 2002

I'un des centres de culture

scientifique français les plus

dynamiques : Cap Sciences.

Créé en 1994, Cap Sciences

est actuellement dirigé par

Bernard Alaux, dont l'énergie

et l'esprit d'initiative habitent

cet espace de partage des

savoirs.
Pour évoquer Cap Sciences, je vois deux métaphores : le pont et la remontée à contre courant.

Le pont. En cours de construction en aval de la ville, le nouveau pont levant de 430 mètres de long décongestionnera d'ici deux ans l'esthétique "vieux pont de pierre " qui donna son unité à la ville de Bordeaux, il y a près de deux siècles. Ce pont BacalanBastide se situe presque en face de Cap Sciences... Occasion pour le musée de faire vivre aux visiteurs les étapes du chantier, mais aussi de faire comprendre les paramètres techniques et scientifiques de la construction d'un pont, objet d'une exposition en temps réel (Secrets de ponts). La métaphore est, bien sûr, celle de la passerelle de savoirs à établir entre les sciences, les techniques et la société. Il est assez remarquable que la revue de Cap Sciences, H2O, se présente comme un magazine des sciences et de l'industrie, ce dernier mot que l'on évoque le plus souvent de façon pudique après sciences et technologie. Mais c'est la volonté affichée de Bernard Alaux de faire une grande arche pour réunir ces deux rives extrêmes de savoirs. Et Bernard a bien su s'ancrer de façon très ferme dans le tissu régional qui fait de Cap Sciences un élément central de l'activité économique et industrielle de la région Aquitaine, débouchant en particulier sur l'emploi de jeunes formés au Centre, en plus du personnel dont l'activité en dépend directement (une centaine).

La remontée à contre courant, à partir de l'estuaire de la Gironde. C'est bien l'ambition d'un retour aux savoirs de base à partir du réel, tout comme le pont dont on examinera les matériaux qui le composent et les structures de la construction. C'est bien l'enjeu des animations scientifiques (L'atelier d'Arthur, les expositions interactives) pour les jeunes et les 15-25 ans en route vers la vie professionnelle. Il y a quelques années, j'avais beaucoup apprécié une exposition sur le sommeil qui a circulé dans toute la France, ou encore Clim'City : en marche pour un monde durable (maintenant devenue Clim'Way). Pendant ma visite, j'ai été séduit (alors que je ne suis généralement pas très fanatique des expositions basées sur l'informatique) par Numériquement vôtre, réalisée avec la collaboration de l'Espace des sciences de Rennes : beaucoup de vraie interactivité, et pas seulement celle derrière un clavier ou devant un écran.

La physique est naturellement présente, en particulier par la photonique, un des thèmes forts de la recherche bordelaise, qui a donné lieu à une exposition : Le laser, un concentré de lumière, et à des interventions régulières dans les lycées et collèges sur l'optique et le laser. Le réseau européen Photonics explorer, auquel est associée une équipe de Cap Sciences, met en place, à l'aide d'un ensemble de prototypes et d'une méthode d'enseignement ludique, des expérimentations visant à renforcer l'attractivité des sciences de base pour les élèves du secondaire.

Comme toutes les autres réalisations, ces expositions, une fois mises au point et présentées à Bordeaux, circulent en région et au-delà. Sur les 220000 visiteurs annuels de Cap Sciences, la moitié des personnes touchées vient d'animations itinérantes. De plus, l'activité et le rayonnement de Cap Sciences le conduit à aider à la mise en place de structures comparables en France (Toulouse, Guyane...). La construction de ce centre de $3600 \mathrm{~m}^{2}$, avec un faible budget total ( $3 \mathrm{M} €)$, la gestion $(30 \%$ de ressources propres!), la qualité des contacts avec la recherche aussi bien qu'avec le milieu économique et social, sont en effet des points de référence pour d'autres réalisations 


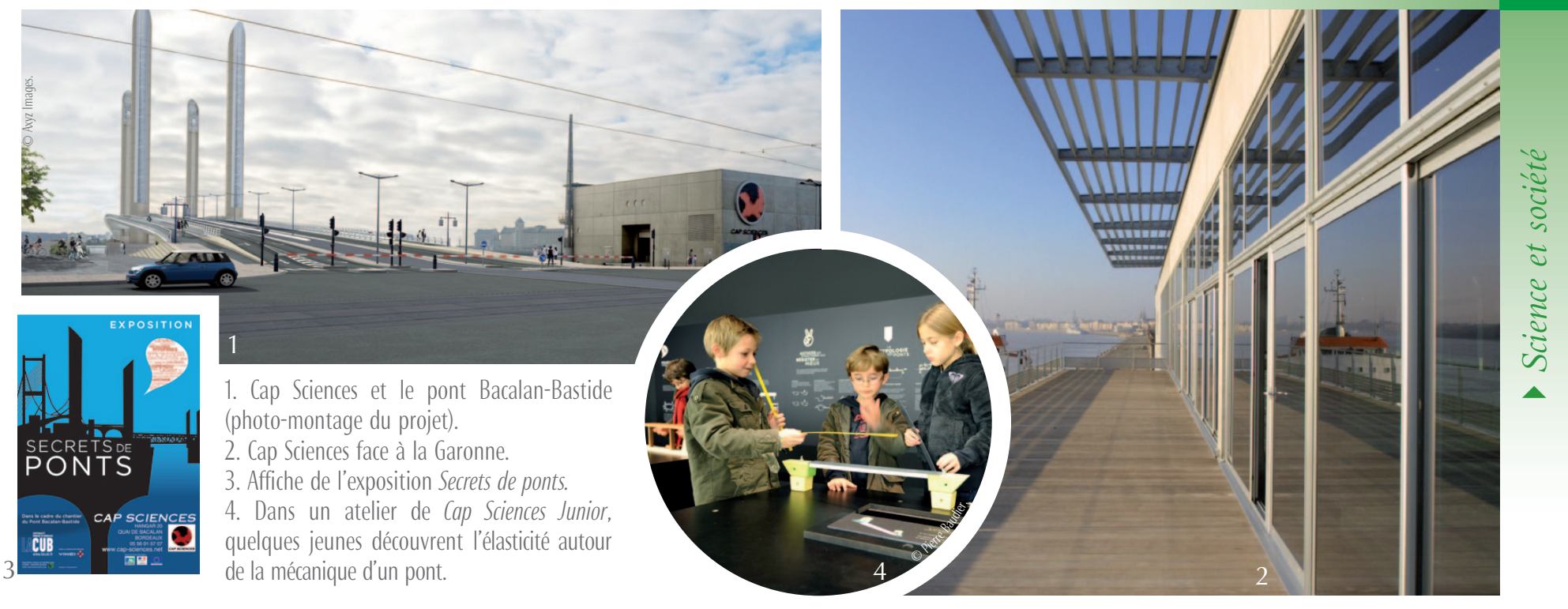

de ce genre, certainement plus que des copies en réduction de nos mégamusées!

Alors que je visitais Cap Sciences, pendant la semaine du Congrès général 2011 de la SFP (qui y avait été accueilli la veille au soir), Bernard Alaux a reçu un appel l'informant qu'il venait d'être retenu pour un soutien national de $15 \mathrm{M} €$ dans le cadre des Investissements d'avenir (le projet INMEDIATS). Le couplage avec l'université de Bordeaux, elle-même reconnue dans le cadre des IDEX (Initiatives d'excellence), est un gage pour l'avenir de la vie culturelle scientifique en Aquitaine.

Revenant de cette visite, je revois l'ultime réunion d'un dernier conseil scientifique commun entre le Palais de la découverte et la Cité des sciences, il y a un peu plus d'un an, juste avant la fusion en Universcience. Avec Philippe Lazar (qui préside la Société des amis du Palais de la découverte) à côté de qui je siégeais, nous n'avions tous deux pas réussi à faire passer le message qu'un réseau culturel scientifique ce n'est pas un grand centre (loin d'ailleurs du milieu scientifique) qui fait passer et diffuse la bonne parole tout autour de lui, comme cela nous était alors présenté, mais que c'est un fin maillage de partenariats scientifiques et économiques, locaux, régionaux, fait de trocs, de conseils...; et que le géant Universcience ne pourra, quelle que soit la bonne volonté de sa direction, se substituer à cela. Tout au plus pourra-t-il récupérer les fruits de ces actions diverses, et ce n'est déjà pas mal! Le fait pour le ministère de la Recherche d'avoir confié le financement national de la culture scientifique à un seul organisme, au lieu de soutenir et de s'appuyer sur toutes ces initiatives dont Cap Sciences est un superbe exemple, est, à mon sens, une grossière erreur.

\section{Cap Sciences : Centre de Culture Scientifique,}

\section{Technique et Industrielle Bordeaux-Aquitaine}

Créé en 1994, Cap Sciences est devenu un élément majeur de l'offre culturelle de la région Aquitaine. En 2010, plus de 220000 personnes ont bénéficié de ses activités.

À partir de son bâtiment clairement identifiable, marquant l'entrée des quais depuis le futur franchissement Bacalan-Bastide, Cap Sciences déploie une présence régionale et nationale sous des formes multiples : expositions, conférences, débats, animations, sites internet, publications, liens avec l'innovation, visites de sites industriels et techniques. Administré par les universités, les organismes de recherche et des représentants du monde de I'entreprise et de I'industrie, Cap Sciences est un outil d'interface entre science et recherche, industrie et société.

Cap Sciences joue un rôle important au sein du réseau national des centres et musées de science (AMCSTI), participe à des instances nationales, diffuse ses expositions en France et à l'étranger, exportant ainsi son image et son savoir-faire. II a obtenu en 2008 le label «Science et Culture, Innovation » du ministère de l'Enseignement supérieur et de la Recherche.

Les actions de Cap Sciences s'articulent autour de plusieurs missions : contribuer à la compétitivité et à l'attractivité de la région Aquitaine, permettre aux jeunes de développer leur goût pour les sciences et faciliter leur orientation vers les métiers scientifiques, favoriser les rencontres entre acteurs économiques et éducatifs, contribuer à la cohésion sociale dans les territoires, augmenter le niveau de culture scientifique et technique des citoyens.

Cap Sciences a développé au Hangar 20 " La galerie Industrie \& Recherche », une vitrine dédiée à la mise en valeur des entreprises et des lieux de recherche de la région Aquitaine. Cet espace, bien identifié et utilisé par les industriels, facilite I'approche par les différents publics de la réalité de l'économie, de l'innovation, des métiers, et des enjeux de la recherche. Il permet la consultation d'informations scientifiques et techniques, la découverte de chercheurs, d'innovateurs et d'entrepreneurs sous forme de portraits, une approche des métiers, la présentation d'un secteur industriel, la préparation de visites de type « tourisme industriel».

Cap Sciences contribue à la nouvelle plate-forme d'information scientifique, technique et industrielle, "Info Sciences Aquitaine » (www.infosciences-aquitaine.net/), et met en place un programme d'évolution vers l'économie numérique : cap-sciences.num .

Cap Sciences édite une revue annuelle de 120 pages, $\mathbf{H 2 0}$, et un trimestriel, $\mathrm{H} 2 \mathrm{O}$, le magazine, tirés chacun à 6000 exemplaires, présentant des dossiers et l'actualité scientifique en Aquitaine.

Renseignements et réservations

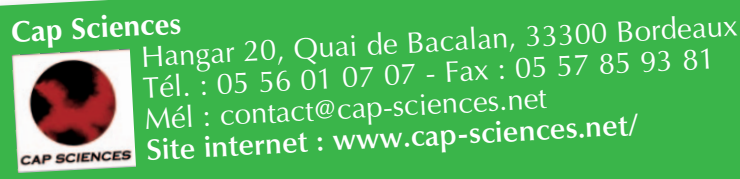

Horaires d'ouverture

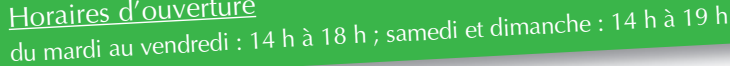

